\title{
L'apport de la civilisation Islamique dans l'héritage de l'humanité
}

\section{Asmaa T. Mohamed ${ }^{(a)}$ Prof. Attia ELKollaly ${ }^{(b)}$}

(a) Assistante à l'Académie des langues internationales (El Madina) à Giza. ${ }^{\text {(b) }}$ Professeur de littérature et de civilisation françaises, Chef du département de français et Vice- doyen pour les affaires de l'enseignement et des étudiants, Faculté des Lettres, Université de Damiette.

\section{Résumé}

La civilisation islamique joue un rôle efficace non seulement dans l'enrichissement de la civilisation occidentale mais encore dans celui de la civilisation humaine. Son apport s'est manifesté dans tous les domaines. En mettant l'accent sur un tel rôle, nous pouvons dissiper le mythe d'exceptionnalisme selon lequel la civilisation occidentale est la meilleure et les musulmans sont en retard parce que l'Islam est une religion qui lutte contre la science. Ce mythe qui est mis en scène pour justifier le racisme antimusulman et la domination par l'Occident de quelques pays islamiques arabes sous le slogan de la modernisation. Une telle civilisation matérielle ne cesse d'inventer les mythes pour le pillage des fortunes des pays vulnérables et par là ne cesse de mettre l'humanité dans l'impasse. Pour sortir de cette impasse, nous essayons de mettre l'accent sur la civilisation islamique.la tâche n'est pas d'élever un mausolée à un mort illustre selon l'expression de Roger Garaudy mais évidemment de savoir comment une civilisation peut voler au ciel de la modernisation par les deux ailes matérielle et spirituelle à la fois. C'est un grand service pour le monde actuel dominé par le matérialisme absolu et le manque du spiritualisme qui "parait insignifiant. Il ne trouve son expression que dans des formes de sectes religieuses qui malheureusement tendent souvent vers le fanatisme. En un mot, en remettant en scène la civilisation islamique, on peut découvrir ce trésor islamique et découvrir le rôle de l'Islam qui ressemble à cette émergence de source de vie qui allait bouleverser le monde Selon la belle formule de Roger Garaudy.

Mots Clés:

Islam Civilisation, Civilisation, Occidentale, Orient Occident, Cordoue Savants Sciences.

\section{Article history:}

Received 6 January 2021

Received in revised form 27 January 2021

Accepted 3 February 2021 


\section{Introduction}

Paresseux, naïfs, barbares,... Ce sont les caractères des musulmans et des Arabes presque depuis le Moyen-Age jusqu'à présent. Cette image fabriquée, monstrueuse et totalement déformée a nourri de nombreux facteurs notamment le media qui joue un rôle considérable et les orientalistes qui contribuent également à cultiver une telle image.

Dans ce contexte, Edward Saïd dit:

"Fort de la conviction, traditionnellement véhiculée par le savoir orientaliste, que les musulmans, des êtres au comportement à la fois puéril et fataliste (Saïd, 2011, p. 107).

Des personnages "puérils et fatalistes' ne sont en fait qu'une partie d'un tout de l'image négative fabriquée par les orientalistes!

L'Occident a ignoré presque totalement le rôle des musulmans ou bien l'apport de la civilisation Islamique dans le rayonnement de la science, cette civilisation qui a brillé sur tout l'Occident.

En effet l'Occident a reçu la civilisation Islamique par l'ignorance d'un côté et par la mutilation ou plutôt la mutilation de l'Autre. Elle se présente comme un danger pour l'Occident.

Huntington dit: "C'est la seule civilisation qui a mis en danger l'existence même de l'Occident" (Huntington, 1997, p. 231).

Donc, la question qui se pose est la suivante: est-ce que les musulmans sont vraiment fatalistes? Et de cette question découle une autre: est-ce que la civilisation Islamique a terrorisé l'Occident?

Roger Garaudy, nous donnant une réponse peut être convaincante, dit: "Il serait d'ailleurs étrange de considérer comme fataliste et résignée une foi qui a conduit les musulmans, en trois quarts de siècle, à renouveler quatre grandes civilisations et à rayonner sur la moitié du monde (Garaudy, 1987, p. 17). 
Donc, la question qui se pose: pourquoi l'Occident ignore-til volontairement les origines orientales en général et la civilisation Islamique en particulier?

On trouve évidemment des écrivains et des auteurs occidentaux caractérisés par la justice et l'intégrité, mais ils sont malheureusement rares. On peut citer par exemple Sigride Hunke.

Garaudy voit que "Le mythe de l'exceptionnalisme grec n'a pu se former que grâce à cette ignorance volontaire ou ce rejet à la fois des origines et de la postérité de l'Athènes de Périclès" (Garaudy, 1981, p. 17) Par ces mots, Garaudy dénonce en fait l'Occident et il tient à rendre hommage à la civilisation Islamique.

\section{Les questions de l'étude}

N'est-il pas temps pour l'Occident, après tant de siècles d'ignorance et de mutilation, de laisser tomber l'idée de l'exceptionnalisme, cette illusion qui depuis longtemps l'entoure?

Garaudy voit que l'Occident, pour laisser tomber le mythe de l'exceptionnalisme et pour reconnaître l'autre Islamique en particulier, il peut se réconcilier avec soi-même et donc il peut découvrir les dimensions humaines et divines perdues dans une civilisation matérielle par excellence. Il dit:

"L'Occident depuis treize siècles, a refusé ce troisième héritage: l'héritage arabo Islamique qui aurait pu et peut encore non seulement réconcilier avec les autres sagesses du monde, mais l'aider à prendre conscience des dimensions humaines et divines dont il s'est mutilé en développant unilatéralement sa volonté de puissance sur la nature et sur les hommes" (Garaudy, 1981, p. 19)

La civilisation islamique a été trouvée non seulement face à la négligence de son mérite mais encore en lutte contre sa rapide propagation!

"A partir du VII siècle, les autorités politiques et religieuses de l'Europe chrétienne développent de multiples stratégies face à l'expansion de la civilisation musulmane dans le monde." (Hajjat \& Mohammed, 2016, p. 164). 
Notre tâche est de mettre en lumière le rôle de la civilisation islamique dans la civilisation mondiale en général et son apport dans la civilisation occidentale en particulier et cela n'est pas facile! Certes, nous consultons tant d'œuvres et d'encyclopédies de langues différentes notamment d'origine arabe et nous les avons traduites en français.

Est-ce que la civilisation Islamique est une nostalgie, une tentative sans but ou un sujet inutile?

Garaudy répond: "En décrivant la trajectoire de l'Islam andalou, notre objectif n'est pas d'élever un mausolée à un mort illustre" (Garaudy, 1987, p. 6).

Donc pourquoi tentons-nous de mettre l'accent sur la civilisation Islamique?

"Pourquoi l'Andalous musulman et sa capitale spirituelle, Cordoue, fut-il le centre de ce rayonnement?" (Garaudy, 1987, p. 6)

Nous allons essayer de répondre à ces interrogations à travers une présentation de l'essor de la civilisation Islamique dans tous les domaines humains.

\section{L'importance de l'étude}

La civilisation islamique joue un rôle efficace non seulement dans l'enrichissement de la civilisation occidentale mais encore dans celui de la civilisation humaine. Son apport s'est manifesté dans tous les domaines. En mettant l'accent sur un tel rôle, nous pouvons dissiper le mythe d'exceptionnalisme selon lequel la civilisation occidentale est la meilleure et les musulmans sont en retard parce que l'Islam est une religion qui lutte contre la science. Ce mythe qui est mis en scène pour justifier le racisme antimusulman et la domination par l'Occident de quelques pays islamiques arabes sous le slogan de la modernisation. Une telle civilisation matérielle ne cesse d'inventer les mythes pour le pillage des fortunes des pays vulnérables et par là ne cesse de mettre l'humanité dans l'impasse.

Pour sortir de cette impasse, nous essayons de mettre l'accent sur la civilisation islamique.la tâche "n'est pas d'élever un mausolée à un mort illustre" (Garaudy, 1987, p. 6) selon l'expression de Roger Garaudy mais évidemment de savoir 
comment une civilisation peut voler au ciel de la modernisation par les deux ailes matérielle et spirituelle à la fois. C'est un grand service pour le monde actuel dominé par le matérialisme absolu et le manque du spiritualisme qui "parait insignifiant. Il ne trouve son expression que dans des formes de sectes religieuses qui malheureusement tendent souvent vers le fanatisme." (ElKolaly, 2001, p. 5)

En un mot, en remettant en scène la civilisation islamique, on peut découvrir ce trésor islamique et découvrir le rôle de l'Islam qui ressemble à "cette émergence de source de vie qui allait bouleverser le monde" (Garaudy, 1981, p. 24) Selon la belle formule de Roger Garaudy.

La civilisation Islamique se distingue par la compatibilité ou plutôt l'harmonisation entre la science et la religion parce que l'Islam encourage la science et honore également les savants.

Dans ce contexte, Garaudy dit: "En Andalous est née, du IX aux XIII siècles lorsque Cordoue (alors la plus grande ville du monde par le nombre de ses habitants et surtout par son Rayonnement culturel) en fut le cœur vivant, une vision du monde, de l'homme et de Dieu, fondée sur une conception plénière de la raison. La science expérimentale en est issue, comme l'a reconnu Roger Bacon, qui la fit rayonner sur toute l'Europe." (Garaudy, 1987, p. 6)

Dans la même ligne d'idées, Sigride Hunke, à son œuvre intitulée le soleil d'Allah brille sur l'Occident, met l'accent sur l'unité entre l'Islam et la science_en disant que cette science brille sur tout le monde. Elle dit: "La science arabe apparaît subitement, à peine passé le 1 er siècle Islamique consacré aux conquêtes et à l'élaboration théologique du Coran. Tel un océan de fleurs, passé la sécheresse hivernale, la science arabe surgit du sol et s'épanouit rapidement à un point qui lui vaut bientôt une renommée universelle." (Hunke, 1997, p. 225).

D'ailleurs, Garaudy résumant comment la science a été encouragée par la civilisation Islamique, dit: 
"Toute cette science s'est développée dans une atmosphère de ferveur religieuse" (Garaudy, 1981, p. 85).

La rupture de la science en Occident avec la sagesse ou la religion met l'humanité dans l'impasse.

Selon Roger Garaudy, "La conception actuelle de la science, le positivisme, cette vision mutilée de la raison, qui fait de la science séparée de la sagesse et de la foi, une fin en soi, et ne pose plus la question du pourquoi?, Du but, la question des limites et des postulats, risque de conduire l'humanité entière au suicide" (Garaudy, 1987, p. 5).

La rupture de la science, de la sagesse et de la religion a engendré tant de questions épineuses comme par exemple le clonage des êtres humains et autres problèmes qui conduisent l'humanité vers l'abîme.

Une autre dimension a été mise en lumière par Garaudy: le danger de la croissance incroyable des armes sur l'humanité, Il dit:

"A Hiroshima, 70000 personnes furent tuées en un instant. Le monde dispose aujourd'hui de l'équivalent d'un million de bombes d'Hiroshima, c'est-à-dire de la possibilité technique d'anéantir 70 milliards d'êtres humains, quinze fois plus que n'en contient de la planète" (Garaudy, 1987, p. 6).

En un mot, on peut dire que "l'Europe(...) est passée de l'ignorance barbare à une barbarie savante." (Garaudy, 1981, p. 75).

\section{L'apport de la civilisation islamique dans différents domaines:}

\subsection{Les libraires}

Le rôle du libraire a été primordial. On peut citer par exemple le libraire Ibn an Nadim. Sigride Hunke dit:

"Le libraire Ibn an Nadim vient de publier à Bagdad son catalogue des sciences. Cet ouvrage en dix volumes relève le titre de tous les livres de philosophie, d'astronomie, de mathématique, de physique, de chimie et de médecine parus Jusqu'alors en langue arabe." (Hunke, 1997, p. 209).

De plus, la bibliothèque de la civilisation Islamique joue un rôle décisif et primordial dans l'enrichissement des universités européennes. 
Sous ce titre "D'où vient l'essor scientifique de l'Europe" (UNESCO, 2010, p. 285)?

On répond: "C'est dans les bibliothèques de Cordoue de Tolède et d'ailleurs que les Occidentaux ont découvert (en arabe) les textes antiques qu'il a d'abord fallut traduire." (UNESCO, 2010).

\subsection{La traduction}

La traduction joue un rôle primordial d'autant plus que les traducteurs prennent une place élevée dans l'Etat. Ils ont été parmi les plus proches des califes et des responsables, ils ont reçu soin et attention et ont gagné beaucoup d'argent pour les services de la traduction ou pour les traductions fournies.

Dans ce contexte, Garaudy dans son œuvre intitulée Promesses de l'Islam, voit que la traduction dans la civilisation islamique prend une position spécial car les traducteurs ont été honorés et encouragés par les califes et cela contribue considérablement à enrichir ce domaine. Donc, les traducteurs jouent un rôle efficace à la vie et ont enrichi la bibliothèque islamique et arabe par plusieurs des ouvrages des langues étrangères traduites en arabe.

D'autre part, l'académie de traducteurs a été inaugurée pour la première fois.

Reste à mettre en lumière les traducteurs célèbres. Certes, Ils sont nombreux. Parmi ces traducteurs, on peut citer les deux les plus célèbres : Ishaq ibn Hunayn et Yaya ben Massoueith le chef de traducteurs qui était aussi un médecin.

\subsection{La médecine}

Selon Sigride Hunke dans son œuvre intitulée, Le soleil d'Allah brille sur l'Occident, au sein des pays arabes, la médecine, reposant sur les bases scientifiques, a vu le jour pour la première fois.

Pour bien comprendre l'influence du médecin et son rôle primordial dans la civilisation Islamique et mondiale, il faut d'abord se rappeler exactement la situation de celui-ci en l'Europe.

Un regard sur la situation de médecin à l'Europe au Moyen Âge.

\section{La médecine et les prêtres.}


Selon Sigrid Hunke, dans son œuvre intitulée Le soleil d'Allah brille sur l'Occident, les prêtres ont le rôle de soigner les maladies. On raconte qu'une fois, un chevalier tombe malade, et quand le prêtre vient le soigner, il met un peu de cire dans le nez du malade! Le malheureux mourut sur le champ!

A ce moment, le prêtre dit: "pour lui épargner de plus grandes souffrances, je lui ai bouché le nez afin qu'il meurt et repose en paix" (Hunke, 1997, p. 119).

Nous allons raconter une autre histoire citée par Sigrid Hunke, dans le même ouvrage, et nous allons la résumer en quelques mots:

Pour soigner un malade qui souffre d'un emplâtre sur la jambe, un médecin franc demande au malade de choisir entre l'amputation de la jambe ou la mort. Le malade choisit de se faire amputer et le médecin lui coupe violemment la jambe et tout à coup le misérable est mort!

\section{Le conte le plus bizarre}

"Le démon l'obsède", Ainsi déclare le médecin quand une femme malade est venue pour être soignée de la fièvre .Donc, le médecin lui coupe la tête par les rasoirs. Tout à coup, la femme a terriblement perdu la vie.

Ce conte est mentionné par Sigride Hunke dans son œuvre intitulée, Le soleil d'Allah brille sur l'Occident, et est raconté par le prince Ibn Mounqidh dans son œuvre intitulée le livre d'al-I'tibar dans un chapitre qui porte le titre "les particularités des francs et leurs morales".

\section{Les malades souffrant des maladies incurables}

Le traitement des malades souffrant de maladies incurables est le suivant: ils ont été enchaînés! Et ils ont été jetés en prison ou dans les maisons ou la tour des fous.

En plus, les malades ont été torturés à mort pour leur arracher le démon du corps!

Selon Sigrid Hunke, "En Espagne musulmane, (...), les malades sont placés dans les hôpitaux, ces derniers portent le nom des hôpitaux des innocents" (Hunke, 1997, p. 119).

L'impact de la médecine Islamique arabe sur celle de l'Occident est considérable. Mais hélas, l'Occident ne rend le 
mérite à la médecine qui est né au sein de la civilisation Islamique.

Dans ce contexte, Sigride Hunke pose ces questions:

"Qui sait que la médecine arabe a imprimé un mouvement décisif à la nôtre le jour où Constantin l'a introduite sur notre sol? Qui connaît le rôle déterminant joué par les Arabes dans le développement de notre médecine"? (Hunke, 1997, p. 193)

Les médecins les plus célèbres sont Ibn Qurra, Ar-Rasi, Ibn Sina, Ibn Al Nafîs.

\section{Ar-Rasi comme exemple}

Selon Sigride Hunke, "Ce n'est pas la civilisation persane qui produit un Rhases ou un Avicenne, c'est la civilisation arabe qui rend les hommes de souche persane capables de faire merveille"! (Hunke, 1997, p. 222)

Par ces mots, met en lumière le rôle de la civilisation Islamique en général et celui du savant et du médecin Ar-Rasi en particulier.

\section{L'importance de L'œuvre d'Ar-Rasi à la médecine}

"Il y a six cents ans, la Faculté de médecine de Paris possédait la plus petite bibliothèque du monde: un seul ouvrage. Et c'était celui d'un Arabe" (Hunke, 1997, p. 137).

Cet Arabe n'est que le médecin musulman le plus célèbre au domaine médical: Ar-Rasi.

La statue à la mémoire d'Ar-Rasi à Paris témoigne du rôle considérable joué par ce grand médecin et sa contribution non seulement à la civilisation Islamique mais encore à la civilisation humaine depuis longtemps jusqu'à nos jours.

\section{Parmi les découvertes,on cite:}

- La petite circulation du sang a été découverte par le médecin Ibn Al Nafîs.

- Les Arabes sont les premiers qui utilisent les moyens aidant à diminuer les ravages de l'infection ou pour réduire le nombre de nouveaux cas, comme par exemple, brûler les choses porteurs les germes pathogènes. Cette nouvelle technique a été adoptée depuis l'ancien jusqu'à nos jours à tout le monde: en Occident comme en Orient. 
$\mathrm{Au}$ dire de Roger Garaudy, "En médecine, l'œuvre culminante fut celle d'Abul Kassim Ezzahraoui (l'Albulcasis des latins, né à Zahra, près de Cordoue en 936). Son traité de chirurgie et de pharmacopée (tasrif) sera traduit en latin à la fin du XII siècle par Gérard de Crémone, publié à Salerne en 1475, à Venise en 1647, faisant ainsi autorité pendant un demi-millénaire" (Garaudy, 1987, p. 8).

En outre, le canon d'Avicenne en médecine est l'un des ouvrages les plus célèbres et les plus considérables, il joue un rôle important dans le domaine de la médecine non seulement au niveau arabe mais encore au niveau mondial.

\section{Les hôpitaux}

Selon Ragheb Sergani,"Les musulmans, à la civilisation Islamique, sont les premiers qui ont créé les hôpitaux au monde" (Serjani, 2009, p. 574).

Les dépenses des hôpitaux Islamiques sont très élevées, par exemple le Mansouri du Caire, l'hôpital le plus grand et le plus célèbre dépensait un million de dirhams par an. Pour autant, ils ouvraient leurs portes gratuitement pour toutes les classes sociales.

\section{Les réformes hospitalières}

$\mathrm{Au}$ dire de Sigrid Hunke,"Les établissements hospitaliers fondés par les califes et les sultans étaient conçus avec un luxe égal à celui de palais royaux" (Hunke, 1997, p. 127).

\subsection{Concernant les chiffres arabes}

Tout le monde connaît aujourd'hui les chiffres arabes. Nous ne pouvons imaginer la vie sans ces éléments. Comment pouvons-nous vivre sans un annuaire du téléphone par exemple? Comment encore ce magnifique monument de sciences et des inventions modernes n'aurait pas pu voir le jour.

Cela explique pourquoi le nom des arabes est inscrit en lettres d'or dans l'histoire sous ce titre "Numération écrite arabe."

Paradoxalement, ils ne prétendent qu'ils inventent ces chiffres, ils ont dit qu'ils les ont redécouvert et ils les nomment "les chiffres indiens" pour rendre le mérite aux indiens. 
Sigride Hunke dit:

"Quant à eux, pourtant, les Arabes n'ont jamais laissé planer le moindre doute sur le fait d'avoir emprunté leur numération aux Indiens" (UNESCO, 2010, p. 40).

\subsection{Concernant le domaine social}

Miskawayh joue un rôle important dans le domaine social. Son livre le tahdhib est d'ailleurs le fruit de travaux:

"Dans le plus important de ses ouvrages, le tahdhib, Miskawayh, insiste fortement sur l'idée que l'homme est un être social et sociable par nature et qu'il a besoin de ses semblables pour atteindre la perfection, savoir le bonheur" (UNESCO, 2010, p. 82).

\subsection{La botanique, la zoologie et la minéralogie}

Selon Montgomery Watt, dans son ouvrage intitulé The influence of Islam on Medieval Europe, les Arabes s'intéressent beaucoup à la classification de types de pierres, d'espèces animales et d'espèces végétales. Les Arabes donnent la priorité aux plantes qui s'utilisent au domaine de la médecine pour fabriquer des médicaments. Ils ont des ouvrages importants en botanique, zoologie et minéralogie.

Le livre des plantes d'Al-Dinawari est le plus célèbre dans ce domaine, un botaniste, historien, mathématicien et astronome kurde, qui mourut le 24 juillet 896 à Dinawar.

Ibn al-Baitar est spécialiste en pharmacologie générale et en particulier en botanique.

4.7 Les mathématiques et l'astronomie (UNESCO, 2010, p. 82)

Les Arabes ont réussi à bien mener les mathématiques et l'astronomie, cela explique l'existence d'innombrables inventions et des réalisations dans ces deux domaines.

On dit que les Arabes s'intéressent beaucoup à l'astronomie pour de multiples raisons surtout pour savoir la direction de la prière. Quant aux mathématiques, cette science a presque été la préoccupation des arabes quand ils ont commencé à faire leurs premiers pas de développement.

Al-Nayrizi, Ibn al-Haytham, Jabir Ibn Aflah, AlKhwarizmi, Maslama al-Mayriti, Ibn al-Saffar, Haly 
Abenragel. Tous ces noms et autres brillent au ciel des mathématiques et de l'astronomie.

Nous allons mettre l'accent sur les œuvres de deux personnages: Al-Khawarizmi et Ibn al-Haytham. Pour l'instant, nous nous arrêtons tout d'abord un peu avec un brillant personnage: Al-Khawarizmi, il est vraiment un génie, il est spécialiste en mathématiques et en astronomie en même temps. Il est doué en mathématiques.

Selon Roger Garaudy, "En astronomie, l'héritage de Ptolémée est intègre et largement dépasse sur tous les plans, ici encore, en unité parfaite avec les fins dernières assignées par l'Islam. L'un des plus grands astronomes du IX, Al Battani (877-918), écrit :"par la science des astres l'homme accède à la preuve de l'unité de Dieu et à la connaissance de la sagesse de son œuvre" (Garaudy, 1981, p. 83).

\subsection{Concernant la Géographie,}

Les musulmans se sont distingués dans de nombreux domaines, particulièrement en géographie.

Al Idrisi qui s'est imposé avec son invention de la carte du monde est le plus célèbre dans ce domaine.

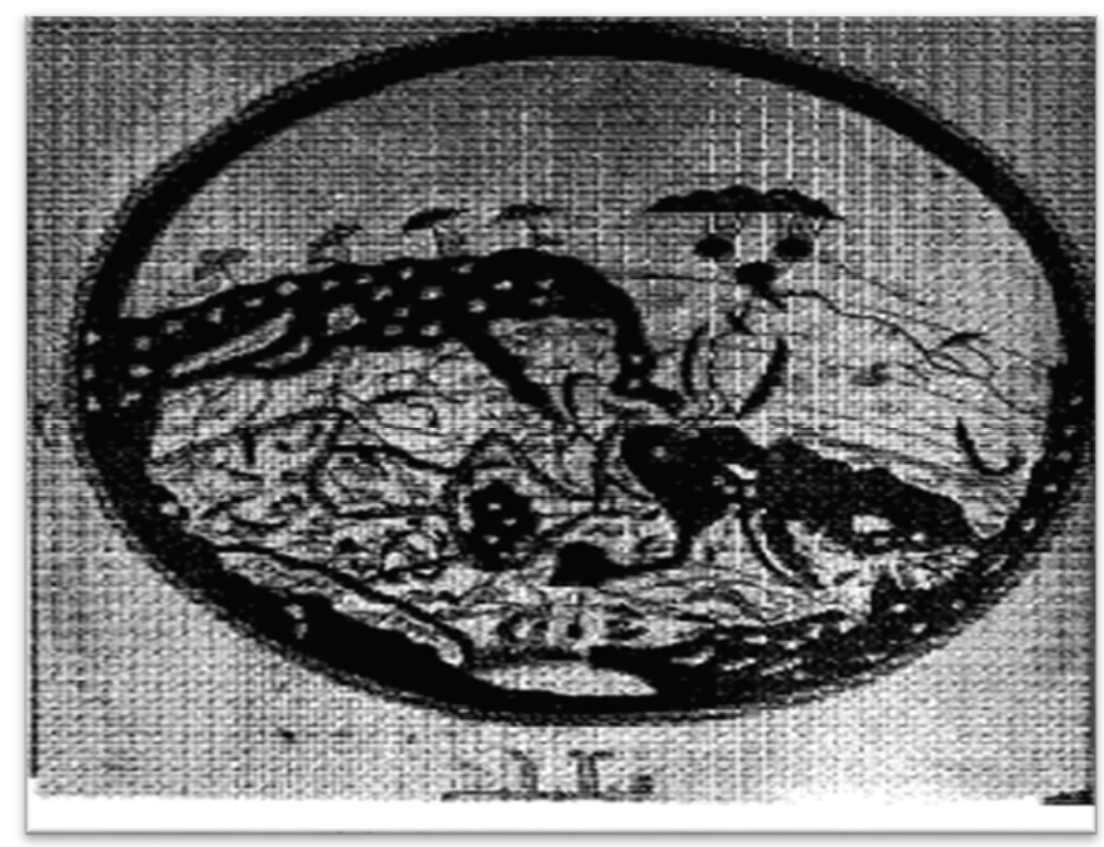

Image (1) La carte du monde Crée par Muhammad Al Idrissi

(Serjani, 2009, p. 286) 


\subsection{L'ingénierie}

Il est étrange peut-être de savoir que l'ingénierie s'est fleurie au sein de la civilisation musulmane.

Le savant et l'ingénieur le plus célèbre dans ce domaine est évidemment Al-Jazari. Il est connu également dans la construction mécanique.

\subsection{Les inventions}

\section{Le Robot, est ce qu'il est une invention ancienne ou moderne?!}

C'est peut être choquant de savoir pour la première fois que le Robot a été inventé depuis longtemps, justement à l'époque de la civilisation Islamique, par un brillant savant musulman: Al-Jazari!

Selon Ragheb Sergani (professeur égyptien à la Faculté de médecine, université du Caire, et un Islamologue), dans son œuvre intitulée, Qu'est-ce que les musulmans présentent au monde, Al-Jazari a inventé le robot pour de nombreuses utilisations et également pour des utilisations utiles comme l'effectuation des choses ou bien le service à la maison.

Donc, le robot joue un rôle considérable à faciliter le quotidien des peuples dans la civilisation islamique.

Récemment, l'Occident se présente le robot comme une invention moderne de la civilisation occidentale qui est exceptionnelle. Cette invention et d'autres accompagnées par une attitude de l'Occident repose sur la supériorité du moi et le mépris de l'autre.

D'ailleurs, nous avons en fait fiers de savoir pour la première fois que le robot et d'autres inventions sont de notre civilisation. Ça nous fait renouer avec notre histoire, notre propre civilisation ou plutôt avec nos mêmes.

\subsection{Les mots d'origine arabe}

L'influence de la civilisation Islamique se manifeste à travers les mots d'origine arabe dans plusieurs langues étrangères. Nous allons mettre l'accent sur quelques mots français d'origine arabe.

\section{Quelques mots français d'origine arabe}

\section{Les boissons}

Sucre, Riz, épinards 
Banane, Abricots, orange

Sorbet, lemonade, Café

Moka, artichaut, cumin

Estragon et safran.

\section{Les vêtements}

Jaquette, mousseline, Satin

Taffetas et Ouate.

\section{Les couleurs}

Carmin

\section{Mots différentes}

Sofa,cafetier, ambre

Tasse, carafe, laque, Tale

Divan, benzine, Borax

Ottomane, alcôve, Soude

Gaze, alcali et camphre.

Dans ce contexte, Le Figaro, dans un article intitulé Connaissez-vous (vraiment) ces mots de l'arabe que vous employez tous les jours?, pose ces questions:

"Que signifie le mot «kawa»? D’où vient le terme «moka»?" "Mais qu'en est-il de «carafe», «tasse» ou bien «douane»?"(1)

Ces questions mettent en scène quelques mots français d'origine arabe.

Donc, Le Figaro nous propose et nous invite de découvrir l'histoire de ces mots français d'origine arabe.

Un tel article montre l'importance de l'apport islamique et arabe dans la vie humaine.

\section{Conclusion}

En un mot, l'apport de la civilisation Islamique, comme nous l'avons vu, dans la civilisation Occidentale s'est manifesté dans tous les domaines. Cela explique pourquoi "Les universités européennes, avec deux ou trois siècles de décalage, celle de Paris comme celle d'Oxford, sont toutes crées à partir du modèle musulman" (Garaudy, 1981, p. 80).

1-https://www.lefigaro.fr/langue-francaise/quiz-francais/connaissez-vousvraiment-ces-mots-de-l-arabe-que-vous-employez-tous-les-jours-20191205, Publié le 5 décembre 2019 à 09:00, mis à jour le 5 décembre 2019 à 16:47. Connaissez-vous (vraiment) ces mots de l'arabe que vous employez tous les jours? 
L'Islam, pour Garaudy, est le sauveur de l'humanité qui est sur le point de naufrage.

Selon lui,"Il (l'Islam) a déjà sauvé de la désintégration de grands empires décadents au VII siècle de notre ère. Peut-il aujourd'hui apporter une réponse à l'angoisse et aux questions d'une civilisation occidentale, qui en quatre siècles, s'est révélée capable de creuser un tombeau à l'échelle du monde (...)?"

Une telle civilisation matérielle qui ne cesse d'inventer les mythes pour le pillage des fortunes des pays vulnérables et par-là ne cesse de mettre l'humanité dans l'impasse.

Il voit que l'Islam porte en lui-même les germes de la vie comme la transcendance et la communauté et avant tout assurément le monothéisme.

En ce qui concerne la transcendance et la communauté, il dit: "Transcendance et communauté, n'est-il pas la contribution que l'Islam peut aujourd'hui apporter à l'invention d'un avenir à visage humain, dans un monde où l'élimination $\mathrm{du}$ transcendant, la destruction de la communauté par l'individualisme et un monde démentiel de croissance ont rendu le statu quo invivable" (Garaudy, 1981, p. 34).

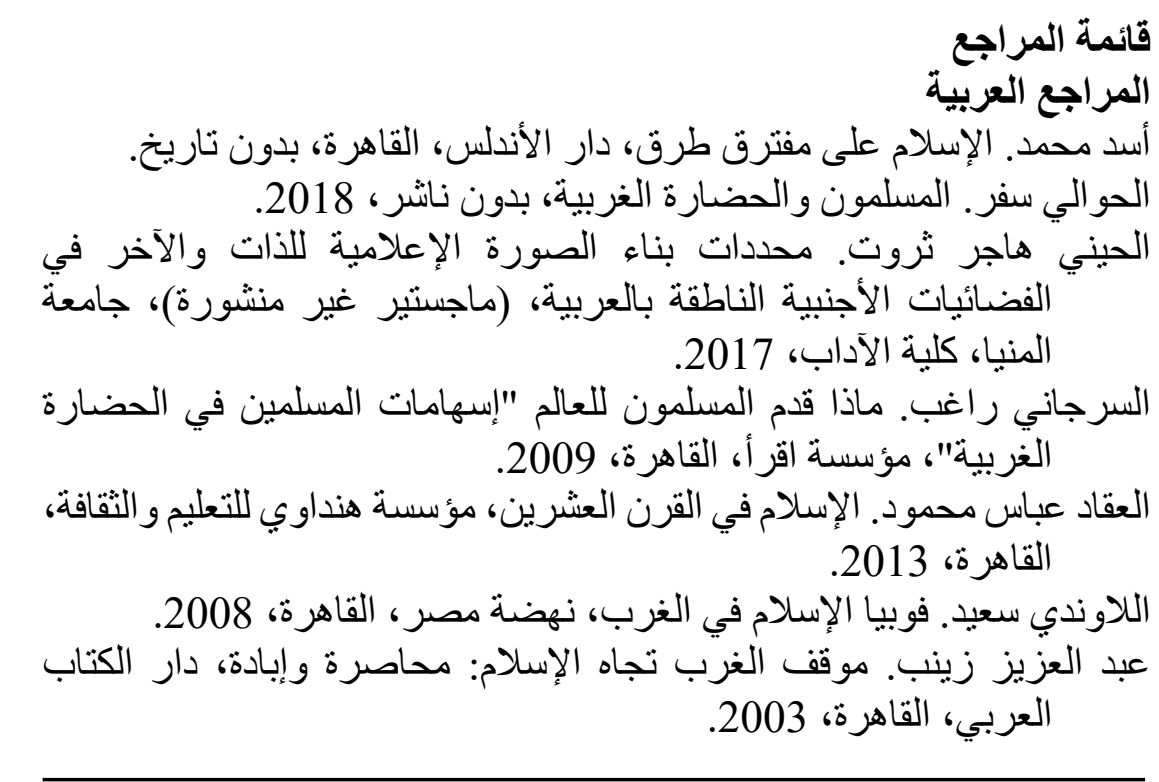


عمارة محمد. الإسلام و الآخر، مكتبة الثروق الدولية، القاهرة، 2004.

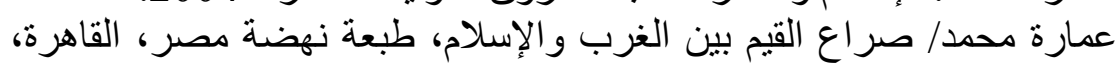
1997 هو فمان مر اد. الإسلام كبديل، مكتبة العبيكان، الرياض، 2002.

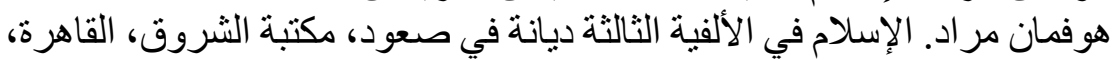
بدون تاريخ.

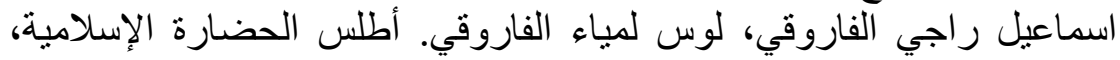
ترجمة: عبد الواحد لؤلؤة، مر اجعة: رياض لون نور الله، ط1. الرياض: مكتبة الرية

\section{Bibliographie}

EIKolaly, A. (2001). le matérialisme au XIX e siècle. Zagazig: Université de Zagazig (thèse de doctorat).

Garaudy, R. (1981). Promesses de I'Islam. Paris: Éditions du Seuil.

Garaudy, R. (1987). L'islam en Occident: Cordoue, une capitale de I'esprit. Paris: Éd. L'Harmattan.

Hajjat, A., \& Mohammed, M. (2016). Islamophobie: comment les élites françaises fabriquent le" problème musulman. Paris: la Découverte.

Hunke, S. (1997). Le soleil d'Allah brille sur l'Occident. éditions Albin Michel.

Huntington, S. P. (1997). Le choc des civilisations. Paris: Odile Jacob.

Saïd, E. (2011). L'Islam dans les medias, traduit de l'anglais par Charlotte Woillez. Paris: Actes Sud.

Serjani, R. (2009). Qu'est-ce que les musulmans présentent au monde: les Contributions des musulmans à la civilisation humaine. I'Establishment d'Iqraa.

UNESCO. (2010). Ia civilisation arabo-musulmane au miroir de I'universel: perspectives philosophiques. Paris: UNESCO. 
المجلة العلمية لكلية الآداب مج 10، ع 1 (2021) 67 - 83

المجلة العلمبة لكلية الآداب

https://artdau.journals.ekb.eg/

\section{إسهامات الحضارة الإسلامية فى التراث الإنسانى}

أسماء ثروت محمد(أ)، أ.د./عطية الإمام القللي (ب).

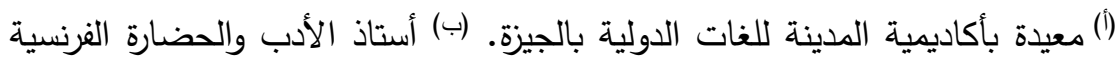

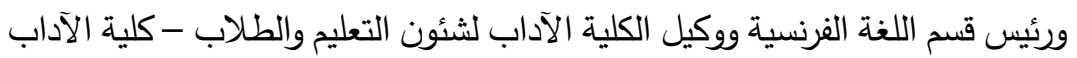

- جامعة دمياط.

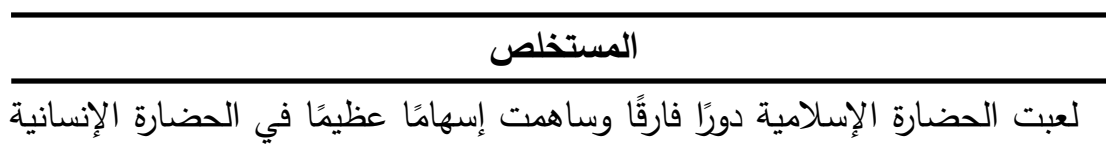

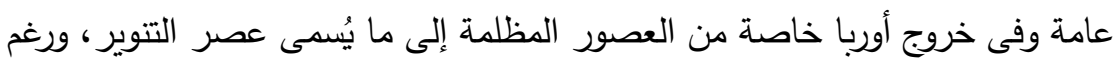

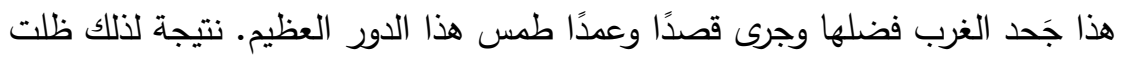

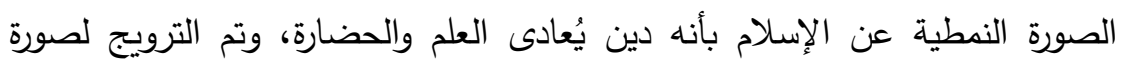

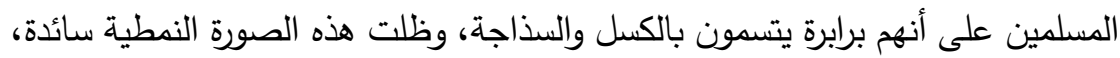

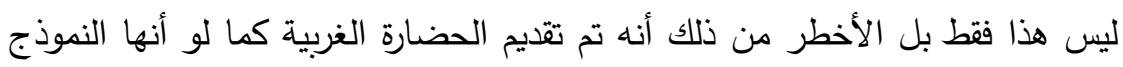
الأمثل والمنقذ للشرق من التخلف الذى يقبع فيه. وتوارت حقيقة هذه الحضارة الغربية التى تسودها المادية المطلقة والخواء الروحي وانهيار القيم، حضارة بتعبير المفكر فئه

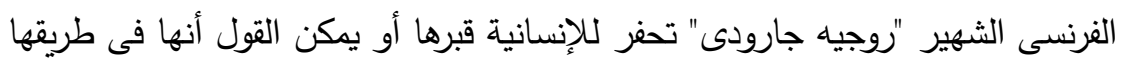

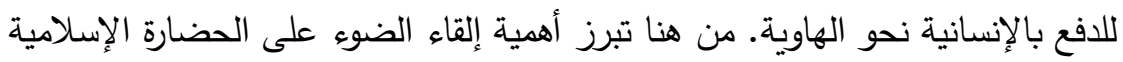

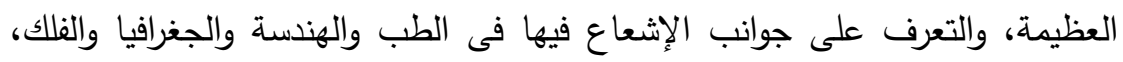

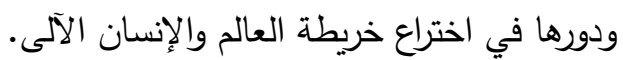
الكلمات المفتاحية: الحضارة الإسلامية، قرطبة، الإلى الحضارة الغربية، الخواء

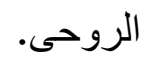

تاريخ المقالة:

تاريخ اســتلام المقالـة: 6 يناير 2021

تاريخ استلام النسخة النهائية: 27 يناير 2021 تاريخ النقاير 2021 تاريخ قبــول الدقالــة: 3 فبراير 2021 